graves de se glisser au travers des gestes que son application réclame. Nous avons devant les yeux trop d'exemples d'infections massives et graves dues à des laits qu'on disait cependant récoltés dans d'excellentes conditions, pour accorder à cette méthode tout le crédit qu'elle mérite apparemment. Nous en revenons donc toujours à la pasteurisation, mais, - est-il besoin de le répéter, - celle-ci ne doit pas être quelconque. Il n'est pas douteux, en y réfléchissant bien, que l'obligation de la pasteurisation entraîne la prise de mesures officielles d'ordre hygiénique. Dès l'instant qu'une ville, par exemple, exige que tout le lait consommé sur son territoire soit pasteurisé, cette ville prend des responsabilités qu'elle doit partager avec l'industriel. Celui-ci doit se soumettre à une surveillance constante. Ce ne sont pas des appareils de pasteurisation encore une fois quelconques qu'il lui sera loisible d'utiliser; les services d'hygiène municipaux auront à intervenir dans leur choix et dans le contrôle de leur marche.

\title{
LES BACTÉRIES THERMOPHILES DU LAIT PASTEURISÉ PAR LA PASTEURISATION BASSE (1)
}

\author{
par
}

\section{ROBERT S. BREED}

Station expérimentale d'Agriculture de l'Etat de New-York, Geneva, N.Y.(2).

La pasteurisation basse du lait (chauffage à $60^{\circ}-62^{\circ} 8 \mathrm{C}$. pendant 30 minutes) a été utilisée sur une très grande échelle pendant les vingt dernières années, aux Etats-Unis. Lorsque ce procédé devint d'un emploi général et que les examens habituels de laboratoire devinrent plus fréquents, les investigateurs notèrent la présence occasionnelle d'un nombre excessif de colonies très petites sur les plaques d'agar préparées avec du lait pasteurisé. Les recherches effectuées pendant ces dix dernières années ont montré que ces petites colonies sont quelquefois produites par la croissance dans les appareils de pasteurisation d'un grand nombre de bactéries facultativement thermophiles qui développent généralement ces petites colonies sur les plaques trop peuplées, ou qui ne trouvent pas la température d'incubation $\left(37^{\circ} \mathrm{C}\right.$.), la réaction ou les aliments dans l'agar (peptone et extrait de viande) suffisamment favorables pour permettre le développement de colonies de dimension normale. On a aussi trouvé, à l'occasion, du lait pasteurisé contenant de grandes quantités de bactéries obligatoirement thermophiles. Ces dernières ne forment pas de colonies sur

(1) Article paru dans le Volume Jubilaire du professeur Ch. Porcher.

(2) J'adresse mes très vifs remerciements à M. C. WoLf pour l'excellence de la traduction. (R. B.) 
les plaques mises à incuber à une température aussi basse pour elles que $37^{\circ} \mathrm{C}$.

La facilité avec laquelle les bactéries thermophiles se développent dans le lait chaud a donné naissance à un véritable problème pour les laitiers qui cherchent à livrer un lait pasteurisé de qualité véritablement supérieure. Le produit obtenu peut contenir un nombre excessif de bactéries thermophiles qui portent atteinte à la qualité du lait dans lequel elles engendrent un excès d'acidité et des saveurs indésirables. Elles peuvent également produire de la présure et être ainsi cause d'un caillage hâtif.

De très petites colonies analogues sont produites sur des plaques d'agar mises à incuber à $37^{\circ} \mathrm{C}$. par eertains streptocoques thermotolérants et par des bacilles non sporogènes qui peuvent survivre, mais ne se développent pas dans du lait à une température aussi élevée que $60^{\circ}-62^{\circ} 8 \mathrm{C}$. Quelques-uns de ces organismes ne produisent pas de grosses colonies, même sur les milieux les plus favorables et sur des plaques non surpeuplées. Quelques-uns ne se développent pas du tout et ils peuvent ne pas apparaître sur des plaques préparées avec les dilutions les plus élevées, car ils ont besoin de la trace de sucre qui est ajoutée avec l'eau de dilution, laquelle contient une petite quantité de lait. D'autres organismes qui ne résistent pas spécialement à la chaleur peuvent aussi produire de petites colonies sur plaques d'agar, mais sont rarement aussi petites que celles déjà mentionnées. Quoiqu'il soit nécessaire de conserver à l'esprit, pendant la présente étude, les organismes qui sont plutôt thermotolérants (résistant à la chaleur) et de se reporter à l'occasion à eux, le but principal du présent travail est d'étudier les rapports des bactéries thermophiles et de la pasteurisation à basse température.

Définition destermes.- On a trouvé qu'une définition des termes: thermophiles et aimant la chaleur qui les limitent aux organismes qui se développeront rapidement à $55^{\circ} \mathrm{C}$. est meilleure qu'une définition qui abaisse cette température à $50^{\circ} \mathrm{C}$. Il y a tout un groupe de bactéries sporogènes mésophiles ( $B$. mesentericus Trevisan, B. subtilis Cohn), de même que certains streptocoques (Streptococcus thermophilus Orla-Jensen, etc. (1919)) et peut-être des lactobacilles qui se développeront jusqu'à $50^{\circ}$ et même $55^{\circ} \mathrm{C}$. En fixant comme limite un développement rapide à $55^{\circ} C$., tous les types de bactéries semblent être éliminés des thermophiles, excepté certaines espèces de bacilles sporogènes et une ou plusieurs espèces d'Actinomyces. Il est douteux que d'autres bactéries que les types indiqués puissent se développer aux températures de pasteurisation habituellement employées en Amérique du Nord (60-62。8 C.).

Quelques-uns de ces types thermophiles montrent une grande 
variété de températures de croissance, de sorte que le terme facultativement thermophile est très' commode à employer pour des organismes dont la température de croissance est aussi basse que $37^{\circ} \mathrm{C}$, d'un eôté, et va jusqu'à $55^{\circ} \mathrm{C}$. ou plus de l'autre. Le terme obligatoirement thermophile est réservé aux thermophiles qui ne peuvent pas se développer à une température aussi basse que $37^{\circ} \mathrm{C}$. Les limites supérieures de croissance pour ces facultativement thermophiles et ces obligatoirement thermophiles sont habituellement entre 70 et $80^{\circ}$ C., quoique quelques-unes montreront une certaine croissance même à quelques degrés au-dessus de $80^{\circ} \mathrm{C}$.

Les termes thermotolérants et résistant à la chaleur sont employés dans le présent travail pour s'appliquer aux organismes qui laisseront au moins $90 \%$ de survivants à une température de $62^{\circ} 8 \mathrm{C}$. pendant 30 minutes et seront incapables de se développer facilement à $55^{\circ} \mathrm{C}$.

Observations antérieures sur la coagulation du lait chaud. - Il est difficile de dire qui fut le premier à observer le fait que du lait maintenu à hautes températures (50 à $70^{\circ}$ C.) se coagulait. Il est tout à fait possible que Nicolas APPERT (1810), qui fut probablement le premier à essayer de mettre du lait en boîtes, puisse avoir trouvé des difficultés lors de la coagulation du lait chaud; ou que Gail BORDEN (qui obtint en 1856 un brevet pour condensation du lait, aux Etats-Unis d'Amérique et en Angleterre) et d'autres, dans leurs expériences antérieures sur Ia condensation du lait, puissent avoir fait la même observation. Il est certain qu'Alexander MÜLleR (1867) observa expérimentalement ce phénomène d'une façon très claire quand il essaya d'acidifier des échantillons de lait à $20^{\circ}, 35^{\circ}$ et $50^{\circ} \mathrm{C}$. Il a consigné ses observations sur l'échantillon $\mathrm{n}^{\circ} 3$ comme suit :

"Dans le cours de la dix-septième heure, le $n^{0} 3$, quoique encore doux, commença à fermenter, de sorte que la plaque de verre fut soulevée par les bulles de gaz qui montaient. Après 20 heures (depuis le début), le $\mathrm{n}^{\circ} 3$ était acide et du sérum jaune verdâtre s'était séparé en grande quantité, de même que dans le $n^{0} 2$; le $n^{0} 1$ ne devint acide qu'à la vingt-troisième heure.

"L'essai ci-dessus montre que le lait s'acidifie d'autant plus rapidement que sa température s'approche plus de la température du sang $\left(37^{\circ}\right)$. Des températures plus basses et plus élevées retardent le développement des ferments acides du lait. Si les températures sont plus élevées, un autre genre de fermentation semble débuter qui mérite d'être étudié plus tard. »

Isolement des bactéries thermophiles. - Un nouveau chapitre de cette histoire commence en 1879 quand MiqueL isola une bactérie sporogène de l'eau de Seine, qui se développait à des 
températures élevées $\left(42-70^{\circ}\right.$ C.). L'organisme fut ultérieurement dénommé Bacillus thermophilus Miquel (1888). Ceci fut le début de recherches de ce bacille ou d'autres bactéries analogues dans une grande variété de produits. Pendant la période entre HüPPE (1884) et FLÜGGE (1894), plusieurs bactériologues essayèrent de stériliser le lait, soit pour l'alimentation infantile, soit pour des milieux bactériens. Pendant leurs études, ils isolèrent du lait chauffé diverses bactéries sporogènes. Parmi celles-ci, il y avait le Bacillus subtilis, le $B$. mesentericus et d'autres, qui se développent à des températures assez élevées, mais aucun type thermophile du lait ne semble avoir été nettement identifié avant 1894 .

GoRINI (1894) signala des types thermophiles isolés du lait. Il décrivit un bacille facultativement thermophile (qui se développait entre $37^{\circ}$ et $50^{\circ}$ C.), qu'il nomma Bacillus lactis thermophilus. LEICHMAN (1894) isola aussi du lait un bacille facultativement thermophile (optimum : 45-50 C.). RABINowitsch (1895) isola du même habitat trois souches obligatoirement thermophiles (aucune ne se développait en dessous de $42^{\circ} \mathrm{C}$.) qui se développaient abondamment entre 54 et $62^{\circ} \mathrm{C}$. (maximum $75^{\circ} \mathrm{C}$.), et qu'elle désigna sous les noms de Bacillus thermophilus 1, 2 et 3.

RAPPORT ENTAE LES BACTERIES THERMOPHILES DU LAIT ET LA pasteurisation basse. - Pendant la période qui s'écoula entre 1894 et la date (1923) à laquelle commença en Amérique du Nord la discussion sur la signification des colonies en " pointe d'épingle» sur plaques d'agar habituelles, il y avait eu de nombreuses observations, les unes, enregistrées (Golden State Milk Products Company, San Francisco, 1912. Voir : Hussong et Hammer 1928; Thalstrup Pedersen 1915. Voir aussi Orla-Jensen, 1921 ; et Ayers et JoHnson, 1923), les autres, pas, sur la coagulation du lait à hautes températures. Ces observations ne font que répéter en les développant l'observation antérieure de MüLuER déjà citée, ainsi que celles de Bernstern (Anonyme, 1893) et d'autres. Cette coagulation fut supposée ou démontrée être causée par la croissance de bactéries thermophiles.

Les bactériologistes chargés du contrôle habituel des appareils de pasteurisation par la méthode des numérations bactériennes étaient très souvent inquiets quand ils trouvaient des chiffres élevés dans le lait pasteurisé. On supposait qu'ils pouvaient être causés par une recontamination pendant le refroidissement et la mise en bouteilles. Enfin, un investigateur qui essayait d'éliminer ces numérations élevées dans deux laiteries de 1'Oregon (JACoBSEN, 1918, 1918a) trouva qu'il y avait autre chose. Dans une série d'essais, il note un échantillon de lait qui avait 187.500 microbes par $\mathrm{cm}^{3}$ avant pasteurisation dans une bouteille à $144^{\circ}$ F. $(6202$ C. $)$ pendant $40 \mathrm{mi}$ - 
nutes, et qui en avait 246.000 par $\mathrm{cm}^{3}$ après pasteurisation, et il en conclut que la qualité de la flore bactérienne du lait cru était en rapport avec la richesse microbienne du lait pasteurisé.

Toutefois, il semble que la première reconnaissance du fait que la croissance des bactéries pouvait réellement avoir lieu dans le matériel de pasteurisation ait été notée à l'occasion d'études effectuées dans une laiterie de Kansas City, Missouri. Pendant la décade antérieure à la publication du rapport de YATES (1923), on avait porté l'intérêt le plus vif à la qualité du lait livré à Kansas City, intérêt visant spécialement les numérations bactériennes des fournitures de lait brut et pasteurisé. Cette discussion devint plus active vers 1920 quand de grandes quantités de très petites colonies apparurent sur des plaques ensemencées avec du lait pasteurisé. Ces très petites colonies reçurent, pendant la discussion, le nom descriptif de "pointes d'épingle». On essayait d'expliquer la eroissance des colonies en "pointe d'épingle "s sur les plaques d'agar par le changement de composition du milieu agar. La guerre mondiale avait amené le remplacement dela peptone de WITTE et del'extrait de bœuf de LIEBIG précédemment employés, par des produits fabriqués en Amérique, et les conclusions auxquelles on arrivait en assimilant les toutes petites colonies trouvées occasionnellement sur les plaques préparées avec du lait cru à celles se développant sur les plaques préparées avec du lait pasteurisé prêtaient un peu à confusion. Toutefois, les renseignements fournis par YaTEs et GLÖveR (1923) montrèrent que dans le cas des colonies bactériennes des plaques préparées avec du lait pasteurisé, il s'agissait de bactéries s'étant développées pendant la pasteurisation. Leur article semble être le premier rapport publié établissant le fait que des bactéries peuvent se développer dans du matériel de pasteurisation du type à débit continu chauffant le lait à $6208 \mathrm{C}$. pendant 30 minutes. Ils indiquèrent aussi que ces organismes se développent sur les parois du pasteurisateur chaud, et que les grands établissements travaillant journellement pendant longtemps étaient plus gênés par ces organismes que ne l'étaient les petites installations fonctionnant moins longtemps.

Aussitôt après la conna1ssance des faits observés à Kansas City, Ayers et JoHsson (1924) prouvèrent une difficulté analogue dans une grande laiterie de Washington, D.C., et le même accident est décrit par P. G. TAYLOR (1924). AyERS et JoHnson reconnurent de suite que le terme de colonie en "pointe d'épingle " était appliqué au hasard, et remarquèrent que les organismes thermophiles du lait pasteurisé se développaient en colonies de taille normale sur des plaques non surpeuplées quand des milieux et des températures convenables étaient employés. En outre, ils reconnurent la différence entre les. types de bactéries formant de petites colonies sur les plaques préparées avec du lait frais et les types de bactéries formant de petites 
colonies sur les plaques préparées avec du lait pasteurisé. Ils trouvèrent que l'organisme principal qui se développait pendant la pasteurisation du lait était un bâtonnet de taille moyenne, Gram positif, non mobile, aérobie, qui se développait bien entre $30^{\circ} \mathrm{C}$. et $65^{\circ} \mathrm{C}$, avec optimum entre $50^{\circ} \mathrm{C}$. et $62 \circ 8 \mathrm{C}$. Ils le nommèrent Lactobacillus thermophilus. Une culture, parmi les 39 étudiées, était d'un thermophile sporogène qu'ils ne considérèrent pas comme significatif. Cette étude montra de nouveau clairement que les bactéries se développaient dans les cuves employées dans la pasteurisation basse de telle sorte qu'après que les cuves eussent été employées 3 fois ou plus, les numérations sur plaques d'agar-standard à $37^{\circ} \mathrm{C}$. de lait pasteurisé étaient de 100.000 à 240.000 par $\mathrm{cm}^{3}$. Une numération effectuée avec une incubation à $50^{\circ}$ donna 628.000 bactéries par $\mathrm{cm}^{3}$; avec une incubation à $37^{\circ}$, on n’obtint que 202.000 unités. Trois cuves étaient utilisées et elles servaient 17 fois par jour.

D'après SwENARTen (1926), on nota à la fin de 1922 la nature distincte des'colonies en "pointes d'épingle» apparaissant sur les plaques d'agar habituelles préparées à l'établissement de contrôle du lait de Baltimore, et on commença à relever des renseignements sur leur présence en janvier 1923. Ici aussi il y eut des erreurs produites par le fait qu'on considérait les types d'organismes produisant de petites colonies sur les plaques préparées avec du lait brut comme identiques à ceux produisant le même type de colonies sur les plaques préparées avec du lait pasteurisé. Les conclusions de ces auteurs étaient basées sur l'isolement de 52 cultures de lait brut et de lait pasteurisé ; 50 de celles-ci furent reconnues être des streptocoques. Il est significatif qu'une des cultures restantes était d'une espèce sporogène.

JoHNSON et ExwORTHY (1924) furent vraisemblablement les premiers de ceux étudiant les colonies en "pointe d'épingle » à reconnaître que les streptocoques trouvés dans le lait pasteurisé étaient résistants à la chaleur et capables de croissance à des températures allant jusqu'à $50^{\circ}$ C. A cet égard, les streptocoques qu'ils étudièrent diffèrent des streptocoques habituellement trouvés dans le lait brut ( $S$. lactis (Lister) Löhnis, S. mastiditis Migula, etc.) et ressemblent aux streptocoques résistant véritablement à la chaleur (S. thermophilus Orla-Jensen, etc. (1919)). Ils notèrent que la dimension des colonies en "pointe d'épingle " produites par les streptocoques n'était pas spécialement augmentée quand les colonies n'étaient pas en très grand nombre sur les plaques.

EcKFoRD (1927), travaillant avec du lait pasteurisé de la fourniture de lait de Baltimore, n'eut pas de difficulté à isoler de véritables thermophiles sporogènes et trouva, probablement pour la première fois dans le lait, un Streptothrix (Actinomyces) thermophile. Elle parvint à isoler des thermophiles de 312 échantillons sur 450 . Cepen- 
dant, ceux-ci ne furent isolés qu'après une opération d'enrichissement effectuée à $55^{\circ} \mathrm{C}$. pendant 24 à 48 heures. Les sporogènes étaient des aérobies tout à fait vigoureux avec quelques rares facultativement anaérobies, et développèrent des spores terminales. Elle identifia trois des huit groupes de cultures : Bacillus aerothermophilus Weinzirl; $B$. thermononliquefaciens Bergey et $B$. thermoliquefaciens Bergey. Elle considéra le Streptothrix comme une nouvelle espèce et lui donna le nom de $S$. thermophilus.

Elle fit l'intéressante observation que quelques-uns des thermophiles obligatoires cultivés primitivement sur extrait de bouillon ensemencé avec du lait ne purent pas se développer dans du lait maintenu à $62^{\circ} \mathrm{C}$. Elle reconnut que les thermophiles pénètrent dans le lait brut pendant la traite et proviennent des aliments, de la poussière, des mains des personnes pratiquant la traite et des matières fécales des vaches. Le rapport entre les bactéries thermophiles et le développement des colonies en " pointe d'épingle " est done reconnu, ainsi que leur aptitude à se développer pendant la pasteurisation.

H. A. HARDing fut un des premiers à reconnaître la forte croissance des bactéries thermophiles pendant la pasteurisation. Il fut appelé en consultation à Kansas City au début des difficultés et eut ultérieurement l'occasion d'observer des développements de difficultés analogues dans beaucoup de villes depuis l'océan Atlantique jusqu'aux rives du Pacifique. Ses conclusions ont été présentées dans une série d'articles, soit rédigés par lui seul (1923, 1931), soit avec son collègue WARD $(1924,1926,1927,1928,1929,1930)$ ou avec d'autres (ADAMS et HARDing, 1924; HUNGERFord et HARDiNG, 1924). D'autres articles écrits à ce sujet peuvent aussi être examinés avee ceux-ci, tels que ceux rédigés par TANNER (1924), TANNER et H. G. Harding (fils de H. A. Harding) (1926), H. G. Hardinct (1927), Palmer et MaCutcheon $(1928,1929)$, A. R. Taylor (1924), WARD (1926). Malheureusement, certains de ces articles sont seulement des résumés de rapports publiés à l'occasion de la réunion annuelle de la Société des Bactériologistes américains et ils ne relatent pas toujours les expériences sur lesquelles sont basées les conclusions. Un de ceux-ci est une thèse inaugurale non publiée qui contient des renseignements très intéressants.

HUNGERFoRD et HARDING (1924) et A. R. TAYLOR (1924) ont donné diverses observations qui appuient la thèse de YaTES et Glover (1923) que les bactéries peuvent augmenter en nombre pendant la pasteurisation et que des bouteilles de lait prélevées à la fin du travail journalier peuvent en contenir un très grand nombre.

HARDING (1923, 1931), WARD (1926) et HARDING et WARD (1927) 
ont insisté sur le fait qu'il est difficile de procéder à des numérations bactériennes comparables, sur du lait pasteurisé contenant des thermophiles, avec l'agar-standard approuvé par l'American Public Health Association (1) (Association américaine d'Hygiène publique) pour être employé dans les recherches officielles ayant trait au contrôle du lait. Ceci provient tout d'abord de ce que la température employée (370 C.) n'est pas la température optimum pour les thermophiles et ensuite de ce que de légères variations dans les températures de l'étuve produisent de grands changements dans les numérations. En outre, tous les thermophiles ne se développent pas bien sur l'agar-standard, et de petites variations dans la réaction ou la composition de l'agar peuvent empêcher ou causer l'apparition en colonies de certaines espèces (voir : Cooledge (1924), pour un cas de cette nature). Des contradictions apparues dans des numérations obtenues d'échantillons similaires ont conduit à de nombreuses controverses, ou tout au moins à des discussions, entre l'industrie et les fonctionnaires du contrôle.

HARDING et WARD (1924, 1926, 1930), ADAMS et HaRding (1924) et HARDING (1931) ont aussi appuyé fortement sur la présence presque générale de bactéries capables de se développer à la température de pasteurisation dans toutes les qualités de lait, depuis la meilleure qualité certifiée jusqu'aux autres qualités de lait brut et pasteurisé. En raison de la difficulté qu'ils eurent à se procurer du lait brut exempt de "germes pouvant se développer aux températures de pasteurisation ", HARDING et WARD (1927) concluent également qu'il y a "tout lieu de supposer que tout au moins dans certaines conditions, la mamelle de la vache peut être à j'origine de certains des germes à qui sont dues les numérations élevées dans le lait pasteurisé ». Il a été montré par les travaux de TANNER et H. G. Harding (1926), Clark et Dougherty (1926), Hansen (1929) et Rogmes et Frazier (1930) que cette conclusion n'est pas justifiée, tout au moins en ce qui concerne les thermophiles, et que des contaminations par des poussières extérieures, qu'on ne peut pas éviter dans une étable à vaches, sont une explication suffisante de la présence de thermophiles dans le lait de qualité supérieure.

HARDing et WARD $(1927,1930)$ pensent que le matériel de pasteurisation est toujours pratiquement ensemencé avec des germes qui se développent rapidement à la température de pasteurisation. En particulier, dans leur article de 1930, ils donnent l'impression que des quantités importantes de bactéries thermophiles sont présentes dans les produits de pratiquement tous les établissements de pasteu-

(1) Standard Methods of Milk Analysis (Méthodes officielles d'analyse du lait). Amer. Pub, Health Assoc., New-York. 
risation, conclusion qui est plus absolue que ne le justifient les faits.

HARDING et WARD (1924) furent parmi les premiers à publier les résultats de numérations de plaques de lait préparées à hautes températures $\left(63^{\circ} \mathrm{C}\right.$.) en comparaison avec des numérations exécutées à des températures plus basses $\left(40^{\circ} \mathrm{C}\right.$.). Ils trouvèrent utile aussi (1928) l'épreuve de réduction du bleu de méthylène effectuée avec une couche d'huile neutre et pratiquée à une température de $63^{\circ} \mathrm{C}$., en ce sens qu'elle permettait de déceler la présence de quantités de thermophiles allant en augmentant pendant la durée de fonctionnement du matériel de pasteurisation.

Palmer et MoCutcheon (1928) donnent quelques bons chiffres comparatifs relatant des numérations bactériennes effectuées à $37^{\circ} \mathrm{C}$. et à $62^{\circ} 8 \mathrm{C}$. d'échantillons en double de lait pasteurisé commercialement, provenant d'un groupe d'établissements de pasteurisation grands, moyens et petits, de Detroit, Michigan. Beaucoup des numérations à $62^{\circ} 8 \mathrm{C}$. sont plus élevées que celles effectuées à $37^{\circ} \mathrm{C}$. Ils indiquent que les organismes capables de développement aux températures de pasteurisation étaient plus abondants dans le lait provenant des grands établissements.

L'année suivante, les mêmes auteurs (1929) indiquent les résultats obtenus avee les numérations bactériennes sur plaques en maintenant le lait à basses températures. Quand le lait pasteurisé en bouteille était conservé pendant des périodes de 6 et 24 heures à $5 \circ 5 \mathrm{C}$., la numération effeetuée à $37^{\circ} \mathrm{C}$. sur la plaque-standard ordinaire était habituellement très réduite.

TYPES DE BACTERIES THERMOPHILES DANS LE LAIT PASTEURISE. A l'excéption de ceux d'AYERS et JoHnson (1924) et d'ECKFord (1927), peu des travaux examinés jusqu'à présent ont porté de l'attention aux espèces de bactéries en question dans le problème en discussion. ClaARKe et Dougherty (1926) décrivent deux types de grands bâtonnets, dont un, thermophile facultatif, forme rapidement des spores, tandis que l'autre est décrit comme un bâtonnet thermophile obligatoire non sporogène. Cet organisme est nettement différent du bacille non sporogène décrit par AYERS et JoHNSON (1924), ainsi qu'il est prouvé par les microphotographies et par le fait qu'il était un thermophile obligatoire. Les auteurs ne semblent pas avoir admis qu'il est quelquefois très difficile de prouver la présence de spores dans les bacilles, surtout dans les bacilles thermophiles se développant dans le lait, et s'appuyèrent, ainsi que le firent Ayers et JoHnson (1924), sur le fait que leurs cultures étaient détruites en 10 minutes à $70^{\circ} \mathrm{C}$. pour établir que ces organismes ne formaient pas de spores. Toutefois, l'organisme ressemblait étroitement à un bacille sporogène, ear ils notent qu' "il est remar- 
quable qu'aucune spore ne put être trouvée dans une quelconque des cultures, après une période de plusieurs semaines ».

Tanner (1924), Tanner et H. G. Harding (1926), et H. G. HARDING (1927) ont étudié les types de thermophiles présents dans nos fournitures de lait commercial. Parmi les véritables thermophiles (eroissance à $55^{\circ} \mathrm{C}$. et au-dessus), ils ne trouvèrent rien d'autre que des bâtonnets sporogènes. Ils en concluent que les bactéries thermophiles, quoique pas nombreuses, sont présentes dans tous les échantillons de lait frais sortant de l'étable. Toutes les 73 eultures isolées étaient des bâtonnets Gram positifs sporogènes qui se développaient bien aux températures de pasteurisation. Plusieurs étaient des thermophiles facultatifs. Leur développement dans le lait était lent, mais produisait habituellement un caillé genre présure avec légère alcalinité.

Rogers et Frazier (1930) discutent la nature des bactéries thermophiles dans le lait en comprenant sous le terme de thermophiles tous les organismes se développant à $50^{\circ} \mathrm{C}$. ou au-dessus, au lieu de la température habituellement adoptée de $55^{\circ} \mathrm{C}$. Ils les décrivent comme un groupe divers qui comprend des streptocoques, des lactobacilles, représentatifs du groupe colon, bâtonnets sporogènes anaérobies et spécialement aérobies. Ils étudient aussi les sources des thermophiles et leur contrôle dans les laiteries, ainsi que les conditions qui existent dans l'établissement de pasteurisation de Washington, D.C., mentionné plus haut.

Dans des articles récents, MUDGE et ThoRWALDSEN (1930, 1930 a, $1930 \mathrm{~b}$ ) ont supposé l'existence de spores invisibles et un cycle de développement pour un bâtonnet thermophile sporogène qu'ils ont trouvé dans un lait pasteurisé d'une laiterie de Californie. Ils indiquent que l'organisme se développe très peu dans les milieux d'agar, de telle sorte que les numérations furent déterminées microscopiquement et par un procédé de dilution employant le lait comme milieu. Les auteurs considèrent que le temps de la génération calculé par eux à 7 minutes et qui est basé sur des numérations exécutées microscopiquement, est trop court pour être vraisemblable, et, comme la technique de dilution indiquait la présence d'un plus grand nombre de bactéries qu'on ne pouvait le démontrer soit par culture, soit par le microscope, ils trouvèrent nécessaire de conclure à la présence de spores invisibles. Ils ont pris des bâtonnets contenant des spores que l'on considère habituellement comme des sporanges pour des spores en état de germination.

En outre, ils utilisent des renseignements donnés par HUNGERFORD et HARDiNG (1924) pour le calcul de la durée de la génération, quoique ces renseignements aient été obtenus, non de cultures pures se développant dans un milieu toujours constant en quantité, mais d'un pasteu- 
risateur continu où les échantillons prélevés à 30 minutes d'intervalle représentaient du lait d'un caractère toujours changeant. Rien dans les renseignements soumis ne nous semble nécessiter la déduction de spores invisibles ou d'un cycle de vie inusité. Tandis que des spores non colorées au bleu de méthylène passent facilement inaperçues dans des préparations de lait colorées au bleu de méthylène, rien n'a été observé dans les travaux exécutés dans notre laboratoire qui indique l'existence d'un assez grand nombre de spores invisibles des dimensions et de la forme indiquées dans les dessins donnés par MUDGE et ThORWALDSEN.

RAPPORT ENTRE LES BACTERIES THERMOTOLERANTES DU LAIT et la PASteurisation basse. - Avant de discuter en détail les travaux exécutés à la Station expérimentale de Geneva, il faut mentionner quatre autres articles qui examinent une autre phase de la question des numérations élevées du lait pasteurisé. DotTerRer (1923) fut un des premiers à noter que là où les producteurs de lait fournissent du lait à forte teneur en bactéries, les numórations du lait pasteurisé peuvent être aussi élevées parce que de fortes proportions de bactéries sont parfois tellement résistantes à la chaleur qu'elles ne sont pas détruites par la pasteurisation. Dans ces conditions, les numérations tendent à suivre les numérations du lait brut. Les numérations furent exécutées d'après le procédé Frost sur petites plaques, car les organismes se développaient mieux sur les plaques préparées de cette façon.

Dans sa discussion, WAY (1925) fait peu de différence entre les types de streptocoques trouvés dans le lait brut et ceux trouvés dans le lait pasteurisé, quoiqu'on reconnaisse en général que les streptocoques du lait pasteurisé sont plus résistants à la chaleur que ceux trouvés dans le lait brut. On pense que l'augmentation des numérations dans le lait pasteurisé est due en grande partie à la rupture des chaînes de streptocoques pendant le pompage du lait.

OrLA-JENSEN (1919), qui a fait des recherches détaillées sur les types de streptocoques résistant à la chaleur dans le lait pasteurisé à $63^{\circ}$ pendant 30 minutes, montre que les types prédominants sont le $S$. thermophilus et le $S$. faecium. Il a également isolé une espèce non sporogène qu'il dénomma Microbacterium lacticum.

FAY $(1926,1927)$ limita son attention aux organismes formant les colonies en "pointe d'épingle" sur les plaques préparées avec de la crème glacée (ice cream) qui ne se développaient pas en étendue, même sur des plaques non surpeuplées. Souvent ils n'apparaissaient pas dans les dilutions élevées qui étaient privées du sucre fourni par la crème glacée dans les plaques faites avec la première dilution. Il indique que le premier des trois organismes isolés est un bâtonnet court faisant fermenter le sucrose qui, dit-il, ressemble morphologiquement 
au Streptococcus lactis. Comme les autres types isolés, celui-ci est thermotolérant, non sporogène, et non réellement thermophile. La description du premier type correspond tellement étroitement au Streptococcus thermophilus qu'il est probable que cet organisme est la cause de la majorité des colonies en " pointe d'épingle ". L'identité des autres types n'est pas aussi nette, mais quelques-uns d'entre eux montrèrent la morphologie variable du $S$. thermophilus et des streptocoques voisins. Les types sporogènes isolés ne furent pas étudiés, car leurs colonies ne restèrent pas de dimension en "pointe d'épingle " sur des plaques non surpeuplées. La restriction que FAX apporte au terme en "pointe d'épingle" en ne l'appliquant qu'aux colonies d'organismes survivant à la pasteurisation qui restèrent de dimension en "pointe d'épingle» même sur des milieux convenables et sur des plaques non surpeuplées provient de ce qu'il n'étudia qu'un nombre limité de types et pas de vrais thermophiles.

Une excellente étude des types de bactéries qui survivent à la pasteurisation à $63^{\circ}$ pendant 30 minutes, a été faite par SEIBEL (1926-1927) sous la direction de HENNEBERG. Il a fait ressortir la résistance à la chaleur des types de streptocoques décrits par ORIAJENSEN (1919) et celle des autres types de bactéries tels que le Microbacterium lacticam ORLA-Jensen, divers microcoques et tétra coques.

NEWMAN (1930) a étudié récemment les streptocoqnes résistant à la chaleur à l'occasion d'un développement de colonies en " pointe d'épinglen sur des plaques préparées avec de la crème pasteurisée. Des colonies similaires apparaissaient sporadiquement dans des échantillons de lait cru prélevés chez des producteurs isolés. Les enquêtes effectuées dans les fermes pour déterminer la source exacte des organismes produisant les colonies en "pointe d'épingle " indiquèrent que des pots à lait, dans lesquels le lait écrémé pasteurisé était retourné à la ferme, étaient très probablement à l'origine des streptocoques résistant à la chaleur. Un nettoyage parfait des ustensiles sous la surveillance des inspecteurs fit disparaître les colonies en " pointe d'épingle» des plaques préparées avec le lait cru envoyé par ces fermes à l'usine de pasteurisation. La conclusion que tous les streptocoques présents étaient d'une seule espèce et que leur résistance à la chaleur était rapidement modifiée par l'influence de leur voisinage n'est pas vérifiée par les faits. Il est plus que probable que des études biochimiques et des cultures auraient montré que plusieurs espèces de streptocoques étaient présentes, et que les variations apparentes dans la résistance à la chaleur étaient produites par des variations dans la résistance à la chaleur des diverses espèces. 


\section{TYPES DE BACTÉRIES THERMOTOLÉRANTES ET THERMOPHILES ISOLÉES DANS LES ÉTUDES EFFECTUÉES A GENEVA (NEW-YORK)}

Dans le premier rapport publié à Geneva à l'occasion d'un développement dans du lait pasteurisé de grandes bactéries en forme de bâtonnet (RoBertson, Yale et BREed, 1926), il fut trouvé impossible, par tous les procédés employés, d'isoler une bactérie thermophile. Cependant, à Ja lumière d'observations ultérieures, on comprit que ce développement eût pu être produit par quelque sporogène thermophile qui ne fut pas isolé, plutôt que par les spores et les bâtonnets qui, vus microscopiquement, étaient morts. Pendant le cours de cette étude, 140 cultures de bâtonnets sporogènes mésophiles furent isolées et identifiées comme suit : 48 Bacillus subtilis Cohn; 29 B. mesentericus Trevisan; 22 B. vulgatus Trevisan ; 21 B. circulans Jordan; 10 B. albolactis Migula; 2 B. laterosporus Ford; $1 B$. panis Migula; 1 B. cereus Frankland; 1 B. mycoides Flügge; 5 non identifiées. Cette liste ressemble énormément aux listes précédentes d'espèces de bactéries sporogènes mésophiles isolées du lait pasteurisé.

Robertson (1923, 1927 a, b, c, d) limita ses études des types de bactéries qui pouvaient être isolés des colonies en " pointe d'épingle ", observées dans les travaux habituels de contrôle du lait dans diverses villes des Etats-Unis, aux types non sporogènes. Des cultures d'espèces thermophiles sporogènes furent fréquemment isolées dans son travail et quelques cultures en furent envoyées à TANNer et H. G. HARding (1926), qui les étudièrent. Aucune culture de types non sporogènes ne fut retenue pour l'étude dès l'instant que $90 \%$ des cellules n'ont pu sur vivre à une température de pasteurisation de $62^{\circ} 8 \mathrm{C}$. pendant 30 minutes. Cinq espèces résistant à la chaleur furent trouvées remplir ces conditions. Elles sont nommées dans l'ordre de leur résistance à la chaleur : Microbacterium lacticum Orla-Jensen, Sarcina lutea Schröter, Streptococcus thermophilus (1) Orla-Jensen, Sarcina rosea Schröter, et Micrococcus conglomeratus Migula.

La sarcine jaune fut isolée le plus fréquemment, quoique le Microbacterium lacticum et le Streptococcus thermophilus furent aussi rencontrées souvent. On ne trouva que quelques cultures de Micrococcus conglomeratus qui eurent $90 \%$ de survivants aux températures de pasteurisation. Beaucoup d'autres cultures de ce dernier et d'autres microcoques montrèrent un peu moins de résistance à la chaleur. Aucune culture correspondant exactement au Lacto-

(1) Robertson indique qu'il est convaincu que les cultures dè cet organisme, qu'il a identifié comme étant le S. thermophilus, auraient dû être identifiées comme S. glycerinaceous, et que les cultures qu'il isola du S. thermophilus furent perdues avant qu'il ne pût en faire une étude biochimique complète (communication personnelle). 
bacillus thermophilus d'AYERS et JoHNSON ne fut trouvée, quoique le Microbacterium lacticum fut estimé très semblable à cette espèce. Cependant, des cultures de cette dernière espèce ne purent pas être amenées à se développer dans du lait écrémé maintenu aux températures de pasteurisation.

Dans le travail de Hucker (1928), une étude fut faite des coecus qui survivent à une pasteurisation à $61^{\circ} 1 \mathrm{C}$. pendant 30 minutes, cette pasteurisation étant effectuée dans des conditions de laboratoire rigoureusement contrôlées. Le lait fut maintenu à $10^{\circ} \mathrm{C}$., $20^{\circ} \mathrm{C}$, et $30^{\circ} \mathrm{C}$, , pendant 4 heures avant la pasteurisation. On trouva que ee traitement préliminaire avait une influence sur les types d'organismes présents, en ce sens qu'il y avait une plus grande prédominance des types résistant à la chaleur dans le lait qui n'était pas refroidi avant la pasteurisation. Cent quatre-vingts races de coccus furent isolées du lait pasteurisé et furent identifiées. Le Streptococcus thermophilus Orla-Jensen fut trouvé être le streptocoque prédominant, quoiqu'on trouva aussi le $S$. faecium Orla-Jensen et le S. liquefaciens Orla-Jensen. Le S. lactis (Lister) Löhnis ne survécut pas.

Soixante-seize des cent quatre-vingts races furent trouvées être des microcoques sans prédominance spéciale d'aucune espèce. Ceux qui furent identifiés comprenaient le Micrococcus epidermidus (Kliger) Hucker, le $\boldsymbol{M}$. candidus Cohn, le $\boldsymbol{M}$. varians Migula, et le M. luteus (Schröter) Cohn. On doit noter que ces cultures représentent plutôt des coques individuels qui survivaient à $61^{\circ} 1 \mathrm{C}$. pendant 30 minutes. PricketT (1928a, p. 28) donne une liste analogue de coques résistant à la chaleur. Le rôle que le microcoque jaune citron joue en rendant difficile la production d'un lait pasteurisé ayant moins de 30.000 microbes par $\mathrm{cm}^{3}$ (sur plaques) est discuté dans un travail qui a paru alors que cette étude était écrite (novembre 1931) : Eglinton R. et YaLe M. W., Milk Plant Monthly, janvier 1932.

Prickett $(1928,1928 a, 1929)$ a étudié les types de bactéries sporogènes du lait véritablement thermophiles. Il les a identifiées sous les noms probables ou suggérés suivants, Bacillus terminalis Migula var. thermophilus Prickett, B. michaelisii Prickett (synonyme probable, $B$. thermophilus aquatilis liquefaciens Michaelis), B.calidus Blau, B. thermoalimentophilus Bergey, B. nondiastaticus Bergey, $B$. calidolactis Hussong et Hammer et B. kaustophilus Prickett. La majorité de ces bactéries du lait véritablement thermophiles furent trouvées aérobies avee spores terminales ellipsoïdes ou cylindriques. Comme des auteurs précédents, il a trouvé le Bacillus subtilis Cohn très commun. Les cultures identifiées sous ce nom se développaient à des températures aussi élevées que $55^{\circ} \mathrm{C}$., mais ne se développaient pas aux températures de pasteurisation. 
Pricketr, comme ECKFord (1927), trouva des Actinomyces thermophiles dans le lait et estima qu'il y en avait plusieurs espèces. Des auteurs précédents (voir : Morrison et TANNer (1922), RoBERTSoN (1927a, p. 19)) avaient fréquemment noté leur origine : sol, excréments, etc.

RECHERCHE HABITUELLE DES BACTÉRIES THERMOPHILES DANS LE lait pasteurise. - Robertson, Yale et Breed (1926), Prickett (1928a), Breed $(1928,1928 a, 1929)$, Breed, Prickett et Yale (1929), et Prickett et Breed (1929) ont trouvé que l'examen microscopique direct du lait était d'une grande valeur pour démontrer la présence de bactéries thermophiles dans le lait pasteurisé, surtout quand il est employé en même temps que des plaques d'agar ayant été mises à l'étuve à 55-6208 C. De nombreuses microphotographies ont été reproduites dảns ces publications. Les bâtonnets sporogènes thermophiles peuvent ou ne peuvent pas montrer de spores (habituellement terminales) dans les préparations du lait originel, et, dans quelques cas (en raison de ce que les spores sont formées rarement dans des milieux ordinaires), il est difficile de déterminer sil'organisme est un Lactobacillus ou un Bacillus. Souvent, les bacilles apparaissent comme des bâtonnets individuels dispersés, quoiqu'on trouve aussi des chaînes et des bâtonnets en masses. Les bactéries se colorent bien au bleu de méthylène, et le fait qu'elles sont en vie est démontré par des ensemencements sur milieux d'agar convenables et leur développement en étuve à hautes températures.

Des frottis microscopiques effectués avec des dépôts de toiles de filtres employés pour filtrer le lait à $60-62^{\circ} 8 \mathrm{C}$., et des dépôts grattés sur la surface du matériel de pasteurisation ont montré quelquefois des nombres extraordinaires de bâtonnets avec ou sans spores. Même les nombres présents dans le lait pasteurisé lui-même quand des échantillons étaient prélevés après que l'établissement avait travaillé pendant quelques heures atteignaient des dizaines de millions par $\mathrm{cm}^{3}$.

Le rapport entre la présence de bâtonnets sporogènes thermophiles et la formation de colonies en "pointe d'épingle" sur agar préparé pour le contrôle ordinaire du lait pasteurisé a été discuté par Prickett et Breed (1929). Il est de toute évidence que les bactéries obligatoirement thermophiles ne forment pas ces colonies sur les plaques de contrôle habituelles. La connaissance du nombre et des types exacts des organismes du type obligatoirement thermophile découla seulement de l'emploi de l'examen microscopique direct du lait combiné avec un développement à haute température (PRICKETT, 1928a). Les colonies formées par les bâtonnets sporogènes thermophiles ne sont pas de dimensions en "pointe d'épingle" quand elles se développent sur des plaques non surpeuplées avec des milieux 
convenables et à des températures favorables; mais avec le milieu et la température employés pour le contrôle habituel du lait en Amérique du Nord, les sporogènes thermophiles facultatifs peuvent former des colonies en "pointe d'épingle" typiques. Les colonies en "pointe d'épingle " sont peut-être, ainsi que l'a indiqué FAY (1927), plus souvent produites par des bactéries thermotolérantes, et les dimensions de ce dernier type de colonies peuvent ne pas êtreinfluencées parle surpeuplement. On croit que les organismes communs formant des colonies du dernier type sont les streptocoques résistant à la chaleur (Streptococcus thermophilus, S. glycerinaceous, etc. Voir : SHERMAN et Stark, 1931). Les colonies en "pointe d'épingle» formées sur les plaques préparées avec du lait pasteurisé sont habituellement très petites en dimension et sont produites par des types de bactéries qui sont, normalement, entièrement différents des types de bactéries formant les petites colonies trouvées quelquefois sur des plaques d'agar préparées avec du lait brut. Ces dernières sont habituellement produites par des streptocoques mésophiles et d'autres types communs de bactéries.

DISTRIBUTION, CONTROLE ET SIGNIFICATION DES BACTERIES THERMOPHILES DANS LE LAIT. - PRICKETT et BREED (1929), ainsi que le fit EckFord (1927), trouvèrent les bâtonnets thermophiles sporogènes dans la poussière et autres produits analogues des granges de laiteries. Ils étaient de la même espèce que ceux trouvés dans le lait pasteurisé. Toutefois, ces bactéries apparaissaient en tellement petit nombre dans les pots de lait de 40 quarts (environ 38 litres), tels qu'ils étaient livrés par les laitiers à un établissement de pasteurisation de Geneva, que l'incubation d'échantillons de $10 \mathrm{~cm}^{3}$ à $62 \circ 8$ C. pendant trois jours ne montra des bactéries thermophiles que dans 26 cas sur les 180 échantillons différents prélevés sur des pots provenant de 60 fermes (Pricketr, 1928a). Voir aussi HARDING et WARD (1924) qui obtinrent des résultats analogues. YALE (1929) et YALE et BreED (1930) trouvèrent des numérations sur plaques d'agar d'échantillons de lait cru prélevés sur la fourniture de laits crus mélangés de grands établissements urbains qui, à $62^{\circ} 8 \mathrm{C}$., ne s'élevaient qu'à quelques rares centaines par $\mathrm{cm}^{3}$, à moins que du lait pasteurisé n'ait été ajouté au lait cru. On suppose que le nombre des bâtonnets sporogènes thermophiles dans le lait cru est rarement, et souvent il ne l'est pas, plus élevé que quelques rares centaines ou milliers par $\mathrm{cm}^{3}$.

Prickett et Breed (1929), Yale (1929), Yale et Breed (1930) ont résumé les conditions du travail des établissements de pasteurisation qui favorisent le développement des bactéries thermophiles. En général, un chauffage prolongé du lait à la température de pasteurisation de $6208 \mathrm{C}$., ou le passage continuel pendant quelques heures 
d'un courant de lait chaud à travers les appareils de pasteurisation produisent des conditions favorables à leur développement. Aucune de ces conditions ne peut être réellement considérée comme antihygiénique.

Les opérations particulièrement fautives connues jusqu'à présent comme favorisant le développement de thermophiles pendant la pasteurisation basse peuvent être indiquées comme suit : 10 repasteurisation $; 2^{\circ}$ maintien prolongé du lait en cuves ou stagnation dans des extrémités coudées de tuyauteries aux températures de pasteurisation; 30 usage continu de cuves ou de récipients à courant continu pendant plus de 2 à 5 heures sans nettoyage des appareils par forte aspersion d̀ l'eau très chaude ; $4^{\circ}$ passage de lait chaud à travers des filtres en tissu pendant plus de 1 à 2 heures; 50 présence d'écume sur le lait, laquelle ne s'écoule pas quand les cuves sont vidées à la fin de chaque période de 30 minutes ; $6^{\circ}$ croissance de thermophiles sur les parois du matériel et dans les résidus de lait sur le matériel.

On n'a pas trouvé de rapport particulier entre la présence de thermophiles et le type de matériel de pasteurisation employé. Le lait peut être chauffé trop longtemps dans n'importe quel matériel et. l'emploi prolongé de n'importe quel matériel sans lavage semble invariablement être la cause des difficultés qui se produisent.

HANSEN (1931) a montré par des expériences d'alimentation qu'aucun des sporogènes thermophiles examinés n'a produit dans le lait de substance causant des dérangements intestinaux chez les lapins ou les cobayes. Un témoignage encore meilleur de ce que ces organismes n'ont aucune importance au point de vue de l'hygiène publique a été fourni au cours des études effectuées par PRICKETT (1928a) et YALE (1929) à Buffalo. Dans un des établissements étudiés, une très grande proportion du lait mis en bouteilles montra un nombre de millions de germes par $\mathrm{cm}^{3}$ du thermophile protéolytique très actif qu'est le Bacillus kaustophilus Prickett. A ce moment, il n'y eut aueune plainte de clients et l'emploi du lait ne donna aucun mauvais résultat. Une situation semblable s'est présentée dans de nombreuses autres villes. On a noté le développement de l'acidité, de la coagulation genre présure et des saveurs mauvaises dans quelquesunз de ces cas, mais aucun témoignage d'un préjudice important au point de vue de l'hygiène publique.

RAPPORT ENTRE LE NOMBRE DE THERMOPHILES ET LA PASTEURISATION A haute temperature, - YaLE (1930) étudia un des nouveaux types de pasteurisateurs rapides à haute température (1620 F. $\left(72^{\circ} 2\right.$ C.) pendant 20 secondes) et fit la découverte intéressante que ce genre de pasteurisation n'était vraisemblablement pas favorable au développement de nombres excessifs de bactéries thermophiles. D'autres études dans ce sens sont effectuées sur d'autres 
types de pasteurisateurs nouveaux qui utilisent des durées et des températures analogues, et un rapport sur cette question paraîtra bientôt dans un bulletin de la Station expérimentale d'Agriculture de l'Etat de New-York, de Geneva. Cette découverte explique probablement pourquoi les investigateurs européens vivant dans des régions où la pasteurisation est effectuée à des températures plus élevées n'ont pas remarqué ce phénomène du développement d'un grand nombre de thermophiles sporogènes à l'occasion de la pastenrisation. On peut supposer avec raison que des conditions analogues à celles étudiées dans le présent travail existent à l'occasion de la pasteurisation en Angleterre et dans les autres pays où la pasteurisation basse est employée; mais autant que nous puissions l'affirmer aueun fait analogue à ceux qui ont été signalés en Amérique du Nord ne l'a été dans la littérature en Europe. Certains auteurs européens ont cependant discuté récemment sur les espèces thermophiles dans le lait de marché (J ANOKE, 1918; GoRINI, 1930, 1931).

\section{GONGLUSIONS.}

L'étude sur la présence de bactéries thermophiles dans le lait pasteurisé par pasteurisation basse a montré que les numérations effectuées sur plaques d'agar lors du contrôle habituel du lait ne sont pas suffisantes pour montrer les eonditions bactériennes réelles du lait pasteurisé. Il est, par conséquent, recommandé d'exécuter en même temps que l'examen sur plaque d'agar des examens microscopiques directs de ce lait. Quand le microscope révèle de grandes bactéries en forme de bâtonnets, cela signifie la présence probable de bactéries thermophiles. Des plaques supplémentaires mises à l'étuve à hautes températures (de préférence à $55^{\circ} \mathrm{C}$.) montreront si cette assertion est correcte. Le développement sur ces plaques de bactéries qui ne se développent pas à $37^{\circ} \mathrm{C}$. révèle la présence de bactéries obligatoirement thermophiles. Les bactéries du lait pasteurisé qui se colorent bien au bleu de méthylène sont habituellement trouvées vivantes. Le fait que des thermophiles obligatoires ne se développent pas sur des plaques mises à l'étuve comme d'habitude n'a pas discrédité, comme l'ont dit quelques auteurs américains, l'emploi de la méthode des plaques d'agar-standard pour le contrôle officiel du lait pasteurisé. Il a toutefois prouvé qu'il était nécessaire de faire preuve de plus de réserve et de plus de connaissances pour utiliser les résultats obtenus sur plaques d'agar dans le contrôle officiel.

Des statistiques dignes de confiance montrent que depuis qu'on utilise les numérations sur plaques d'agar dans le contrôle habituel du lait en Amérique du Nord (1900 à 1905), ce genre de travail de laboratoire s'est développé avec une énorme rapidité. En 1929-1930, 
les laboratoires d'hygiène des Etats, des provinces et des villes des Etats-Unis et du Canada ont examiné plus de 567.000 échantillons de lait par la méthode des plaques d'agar. Les répartiteurs de lait de ces régions ont aussi trouvé que cette méthode était pour eux d'une valeur suffisante pour déterminer la qualité du lait commercial qu'ils vendent et pour déterminer aussi les primes payées aux laitiers, puisqu'ils ont examiné un total de plus de 2.105.000 échantillons par la même méthode pendant cette même période. Ce contrôle continue à se développer rapidement, malgré les complications introduites par la croissance rapide de thermophiles sporogènes pendant la pasteurisation du lait à basse température.

\section{OUVRAGES CITÉS.}

F. O. AdAms et H. A. HARDING : 1924. Observations on thermophilic bacteria in milk from farms. Abst. Bact., 8, 18.

Anonyme : 1893. Zur Versorgung grosser Städte mit frischer Milch. Milch-Zeitg., 22, 259-260. (Le résumé de l'ouvrage de Bernstein est donné.)

Nicolas APPERT : 1810. The art of preserving animal and vegetable substances for many years. XV +113 , Patris \& $C^{i e}$, Quai Napoléon, Paris. Traduit par K. G. Bitting, Chicago, Ill., 1920.

S. H. Ayers et W. T, JoHnson junior: 1923. The problem of transporting milk in hot condition. Jour. Dairy Sci., 6, 608-615.

1924. Studies on pasteurisation. XII. Cause and significance of pin point colonies from pasteurized milk. Jour. Bact., 9, 285-300.

R. S. BREED : 1928. Heat-resistant and heat-loving bacteria in their relation to the pasteurization of milk. New York Agr. Exp. Sta. Bull., 559, 19 pages,

1928a, Heat-resistant and heat-loving bacteria in pasteurized milk. Proc. Intern. Assoc. Milk. Dealers, 21st Ann. Conv., 16-35.

1929. Thermophiles in pasteurized milk and their relation to certain types of equipment. Proc. Intern. Assoc. Milk Dealers, 22nd Ann. Conv., 5-33.

R. S. Breed, P. S Prickett et M. W. YALE: 1929. The significance of thermophilic spore-forming bacteria in pasteurized milk. Jour. Bact., 18, 37-38.

J. J. ClaArke et J. M. Dougherty : 1926. A study of the thermophilic bacteria in pasteurized milk. Dissertation pour le grade de Docteur à l'Univ. Cath. d'Amér., Wastington, D.C., 1926.

L. H. Cooledge : 1924. A probable explanation of high count (pin point colonies) in pasteurized milk. Abst. Bact., 8, 20.

W. D. Dotterker : 1923. Some observations on high counts in milk freshly pasteurized unde, commercial conditions. Intern. Assoc. Dairy and Milk Inspectors, 12th Ann. Rpt., 204-214.

Martha O. EckFord : 1927. Thermophilic bacteria in milk. Amer. Jour. Hyg., 7, 201-221.

A. C. FAY : 1926. Thermotolerant saccharophilic organisms as a cause of "pinpoint" colonies in the bacteriological analysis of ice eream. Jour. Bact., 11, 96-97. 
1927. Thermotolerant organisms as a cause of so-called "pin-point" colonies. Jour. Bact., 13, 347-377.

C. FLÜGGE : 1894. Die Aufgaben und Leistungen der Milchsterilisirung gegenüber den Darmkrankheiten der Säuglinge. Ztschr. f. Hyg., 17, 272-342.

C. GoRINI : 1894. Studi critico-sperimentali sulla sterilizzazione del latte. Giornale della reale Societa d'Igiene, 16, 5-24.

1930. Bactéries acidoprotéolytiques dans le lait pasteurisé. Compt. Rend. de l'Acad. d. Sc., Paris, 191, 885-886.

1931. Acidoprotéolytes et thermophiles dans la pasteurisation du lait. Le Lait, 11, 225-233.

P. Arne Hansen : 1929. VII. The udder as a possible source of thermophilic bacteria. New York Agr. Exp. Sta. Tech. Bull., 158. Abst, in Jour. Bact., 19, 46, 1930.

1931. Thermophilic bacteria in milk pasteurized by the holding method. Intern. Dairy Cong. Copenhagen, Conference Papers, Sect. II, Chem. Bact. Hyg., 10-19.

H. A. HARDING : 1923. Sources of variation in bacterial count of pasteurized milk. Abst. Bact., 7, 23-24.

1931. Do thermophilic bacteria in milk rob plate counts of meaning ? Food Industries, 3, 290-292.

H. A. HARDING et A. R. WARD : 1924. Thermophilic bacteria in composite samples from milk plants. Abst. Bact., 8, 19.

1926. Thermophilic bacteria and milk pasteurization. Jour. Bact. 11, 95-96.

1927. What are the sources of high bacterial counts in pasteurized milk? Intern. Assoc. Dairy and Milk Inspectors, 16th Ann. Rpt., 101-110.

1928. Conducting the methylene blue test at $145^{\circ}$ F. Intern. Assoc. Dairy and Milk Inspectors, 17th Ann. Rpt., 289-298.

1929. The bacterial flora of pasteurized milk. Jour. Bact., 18, 35-36.

1930. Observations on effects of thermophilic bacteria in pasteurized milk. Intern. Assoc. Dairy and Milk Inspectors, 19th Ann. Rpt., 135-143.

H. G. HARDING : 1927. The occurrence and significance of thermophilic bacteria in milk. Thèse de Doctorat. University of Wisconsin. Madison, Wis.

G. J. Hucker : 1928. Studies on the Coccaceae. VIII. A study of the cocei resisting pasteurization temperatures. New York State Ágr. Exp. Sta. Tech. Bull., 102. Résumé in Jour. Bact., 15, 28-29, 1928.

F HÜPPE : 1884. Untersuchungen über die Zersetzungen der Milch durch Microorganismen. Mittheilungen $K$. Gesundheitsamte (Berlin), 2, 309-371.

J. D. HUNGERFORD et H. A. HARDING : 1924. Influence of the period of operation of the pasteurizer upon the bacterial count of milk. Abst. Bact., 8, 17.

R. V. Hussong et B. W. Hammer : 1928. A thermophile coagulating milk under practical conditions. Jour. Bact., 15, 179-188.

George J. JACOBSEN : 1918. Unusual factors influencing the efficiency of pasteurization. Abst. Bact., 2, 215.

1918a. Unusual factors influencing the efficiency of pasteurization. The Milk Dealer, 7, mai 1918, 18-20,

F. JANCKE : 1928. „, Thermophile "Bakterien in Milch; Beiträge zur Biologie der „, Thermophilen “. Milchw. Forsch., 6, 303-350.

S. R. JoHnson et Alice Exworthy : 1924. Thermophilic streptococei isolated from milk and their relation to pin-point colonies. Abst. Bact., 9, 24. 
G. LeidhmanN : 1894. Uber eine schleimige Gärung der Milch. Landwirtsch. Versuchs-Sta., 43, 375-398.

P. Mrquel : 1879. Bull. de la Statistique municipale de Paris, décembre 1879.

1881. Etude générale sur les bactéries de l'atmosphère. Annuaire de l'Observatoire de Montsouris. 374-510.

1888. Monographio d'un bacille vivant au delà de $70^{\circ}$ centigrades. Ann. de Microg., 1, 4-10.

L. E. Morrison et F. W. TANner: 1922. Studies on thermophilic bacteria. I. Aerobic thermophilic bacteria from water. Jour. Bact., 7, 343-366.

C. S. Mudge et M. L. Thonwaldson: 1930. Studies on thermophiles in milk. Jour. Bact., 19, 47-48.

1920a. A life cycle of a thermophilic organism. Proc. Soc. Exp. Biology and Medicine,, 28, 202-203.

1930b. Thermophilic bacteria: A problem. Monthly Bull. State Dept. of Agr. California, 19, 710-718.

Alexander Müller : 1867. Chemische Untersuchungen auf dem Gebiete der Milehwirtschaft Landwirtsch. Versuchsstat, 9, 140-141.

R. W. NEWMANN : 1930. Thermoduric streptococei in a dairy area of central California. Monthly Bull. Stxte Dept. Agr. of California, 19, 704-709.

S. Orla-Jensen: 1919. The lactic acid bacteria. Mém. de l'Acad. Roy. des Sci. et $d$. Lettres du Danemark. Copenhague, Sec. d. Sei. 8 sér., 5, 2, 81-196, $51 \mathrm{pl}$.

1921. La pasteurisation du lait. Le Lait, 1, 105-112, 177-183.

R. R. Palmer et C. T. McCutcheon : 1928. Effect of incubation at $145^{\circ}$ F. on bacterial plate counts of milk. Intern. Assoc. Dairy and Milk Inspectors, 17th Ann. Rpt., 279-288.

1929. The effect of holding milk at low temperatures on bacterial plate count. Intern. Assoc. Dairy and Milk Inspectors, 18th Ann. Rpt., 130-135.

P. S. Prickett : 1928. Thermophilic organisms in milk. Jour. Bact., 15, 28.

1928a. Thermophilic and thermoduric micro-organisms with special reference to species isolated from milk: V. Description of spore-forming types. New York Agr. Exp. Sta. Tech. Bull., 147, 58 pages.

1929. Thermophilic spore-formers associated with milk. Jour. Bact., 18, 36.

P. S. PRIOKetT et R.S. BReED : 1929. Bacteria that survive and grow during the pasteurization of milk and their relation to bacterial counts. New York Agr. Exp. Sta. Bull., 571, 25 pages.

Lydia Rabinowitsch : 1895. Uber die thermophilen Bakterien. Ztschr.f. Hyg., $20,154-164$.

A. H. Robertson : 1923. A preliminary report on some non-sporulating, heat resistant organisms in pasteurized milk. Abst. Bact., 7, 357.

1927a. Thermophilic and thermoduric micro-organisms, with special reference to species isolated from milk. I. Review of literature. New York State Agr. Exp. Sta. Tech. Bull., 130.

1927b. II. The thermal resistance of micro-organisms. Vermont Agr. Exp. Sta. Bull. 274.

1927c. III. Description of the non-spore-forming, thermoduric organisms isolated. New York State. Agr. Exp. Sta. Tech, Bulh, 131.

1927d. IV. Effect of age of culture on the heat resistance of non-spore-forming bacteria. Vermont Agr. Exp. Sta. Bull., 275. 
A. H. Robertson, M. W. Yale et R. S. Breed : 1926. Non-thermophilic, spore-forming bacteria associated with pasteurizing equipment. New York Agr. Exp. Sta. Tech. Bull., 119, 11 pages.

L. A. Rogers et W. C. Frazier : 1930. Significance of thermophilic bacteria in pasteurized milk. Amer. Jour Pub. Health, 20, 815-819.

Ernst Seibel et Henneberg, W. : 1926-27. Hitzefeste Bakterien in der bei $63^{\circ}$ C. $\frac{1}{2}$ Stunde, ,dauerpasteurisierten "Milch. Milchwirtsch. Forschungen, 3, 309-312, 4, 41-79.

J. M. Sherman et Pauline Stark : 1931. Streptococi which grow at high temporatures. Jour. Bact., 22, 275-285.

J. C. Swenarton : 1926. Observations on "pin point colony" organisms in the Baltimore milk supply. Jour. Bact., 11, 285-292. Résumé in Abst. Bact., 9, 23-24, 1925.

F. W. TANnER : 1924. Thermophiles from milk. Abst. Bact., 8, 18.

F. W. TANNER et H. G. HARDING: 1926. Thermophilic bacteria from milk. Centralbl. f. Baleteriol., 11 Abt., 67, 330-347. Résumé in Jour. Bact., 11, 96, 1926.

A. R. TAYLOR : 1924. Observations on the increase of the bacterial count during the pasteurization process. Abst. Bact., 8, 17.

G.B. TAYLOR : 1924. Some observations on the seientific control of milk plants from the standpoint of bacteria. Intern. Assoc. Dairy and Milk Inspectors, 13th Ann. Rpt., 287-291.

A. R. WARD : 1926. What ails bacterial counts ? Heat-resisting bacteria. Dairy Products Merchandising, 7, 27-29, 32-36.

H. O. WAY : 1925. Some heat-resisting streptococei found in market milk. Intern. Assoc. Dairy and Milk Inspectors, 14th Ann. Rpt., 179-183.

M. W. YALE : 1929. VI. The control of bacteria that grow during pasteurization. New York Agr. Exp. Sta. Tech. Bull. 156, 25 pages.

1930 The control of thermophilic bacteria in pasteurizing plants. Jour. Bact., 19, 46-47.

M. W. YALE et R. S. BREED : 1930. The control of thermophilic bacteria in pasteurizing plants. Amer. Jour. Pub. Health, 20, 1192-98.

J. W. Yates : 1923. Pin point colonies in plates from pasteurized milk. Abst. Bact., 7, 24.

J. W. Yates et J. J. Glover : 1923. The pin point colonies observed in the bacterial examination of milk, their resistance to heat and growth in different culture media. Intern. Assoc. Dairy and Mille Inspectors, 12th Ann. Rpt., 252-261.

(Traduction de C. WoLf.) 